Artigo de Revisão

Review Article

\title{
Biomecânica aplicada ao polo aquático: revisão de parâmetros cinemáticos e cinéticos da pernada em eggbeater
}

\section{Biomechanics Apllied to the Water Polo: Review of Kinetics And Kinematics Parameters of the Eggbeater Kick}

\author{
Luana Maciel da Silva ${ }^{1} \mathrm{MS}$; Flávio Antônio de Souza Castro $^{1 \S} \mathrm{PhD}$
}

Recebido em: 14 de março de 2016. Aceito em: 13 de junho de 2016.

Publicado online em: 29 de setembro de 2016.

\section{Resumo}

Introdução: 0 eggbeater é uma técnica propulsiva fundamental para o polo aquático. Implica em força resultante propulsiva para cima, sendo utilizada no polo aquático para passes, marcação e chutes a gol.

Objetivo: 0 objetivo desta revisão de literatura foi apresentar e discutir parâmetros biomecânicos (cinemáticos e cinéticos) relacionados à técnica de execução da pernada de eggbeater.

Métodos: As palavras-chave utilizadas para procura dos estudos revisados neste artigo foram: eggbeater kick, water polo e technique, em inglês e português. Foram utilizadas as bases Scopus, Portal Periódico da CAPES e Google Acadêmico. Dezenove estudos foram selecionados para a revisão.

Resultados e Discussão: 0 movimento é caracterizado por uma

Pontos-Chave Destaque

- Definição e descrição do movimento do eggbeater.

- Revisão dos parâmetros biomecânicos do eggbeater. - Classificação quanto à técnica de execução do eggbeater. ação cíclica e alternada dos membros inferiores, ou seja, enquanto a perna esquerda move-se no sentido horário, a perna direita move-se no sentido anti-horário. Estudos demonstram que o movimento não deve ser focado na direção vertical, mas na horizontal (sentido ântero-posterior e médio-lateral) para gerar maior força de sustentação. Em relação à cinemetria, a análise tridimensional parece ser a melhor ferramenta. Já para análise cinética, existe a necessidade de desenvolver tecnologias adequadas à mensuração das forças propulsivas no meio aquático. Ainda, resultados indicam que testes específicos, ou seja, o movimento executado em ambiente aquático mais próximo da realidade de jogo, apresentaram-se mais adequados para avaliar aspectos cinéticos e cinemáticos do movimento em análise.

Conclusão: A fim de se incrementar a força de sustentação, a técnica deve ser horizontalizada. Desenvolvimento de avaliações específicas devem ser estimuladas.

Palavras-chave: polo aquático, técnica, sustentação.

\section{Abstract}

Introduction: The eggbeater kick is a elementary propulsive technique for the water polo.

Objective: The aim of this study was to review the biomechanical parameters (kinematics and kinetics) related to the eggbeater kick, description and analysis.

Methods: The keywords used to search for the studies reviewed were: eggbeater kick, water polo and technique, in English and Portuguese. Scopus, CAPES and Google Scholar bases were used.

\section{Keypoints}

- Definition and description of the eggbeater kick movement. - Review of the biomechanical parameters of the eggbeater kick.

- Classifying the eggbeater kick execution technique.

\footnotetext{
${ }^{\S}$ Autor correspondente: Flávio Antônio de Souza Castro - e-mail: souza.castro@ufrgs.br

Afiliações: ${ }^{1}$ Grupo de Pesquisa em Esportes Aquáticos - Universidade Federal do Rio Grande do Sul
} 
Results and Discussion: The movement is characterized by a cyclic and alternating action of the lower limbs, i.e., while the left leg moves clockwise, the right leg moves in the counterclockwise direction. The eggbeater kick technique promotes a propulsive resultant upward force and it's used in water polo for passes, marking and shots on goal. Studies have shown that the movement should not be focused in the vertical direction, but horizontally (anteroposterior and mid-lateral) to generate more lift. Regarding to kinematics, the threedimensional analysis seems to be the best tool. As for kinetic analysis, it is necessary to develop appropriate technologies to measure the propulsive forces in water. Therefore, results indicate that more specific tests, performed in the aquatic enviroment, next to the game's reality, are more adeqate to asses kinetc and kinematic aspects of the analyzed movement.

Conclusion: In order to increase the lift force, the technique should be horizontalized. Development of specific assessments should be encouraged.

Keywords: water polo, technique, lift.

\section{Biomecânica aplicada ao polo aquático: revisão de parâmetros cinemáticos e cinéticos da pernada em eggbeater}

\section{Introdução}

Dentre os esportes aquáticos, o polo aquático é um esporte coletivo e de invasão praticado em piscinas fundas. Ao passo que é realizado em águas profundas, se faz necessária a utilização de técnicas de sustentação pelos praticantes. O eggbeater, técnica realizada no polo aquático com a finalidade de sustentação e deslocamento, é considerado uma das habilidades fundamentais da modalidade $(1,2)$. É utilizado para elevar a parte superior do corpo para fora da água, gerando força vertical para cima, juntamente com o empuxo, a fim de equilibrar e/ou superar o peso corporal.

O movimento é caracterizado por uma ação cíclica e alternada dos membros inferiores, ou seja, enquanto a perna esquerda move-se no sentido horário, a perna direita move-se no sentido anti-horário, assim, ambos os pés e pernas desenham um círculo (3). Divide-se em duas fases: uma chamada de out-kick e outra chamada de in-kick. Na fase de out-kick os pés e pernas movimentam-se para fora e na fase de in-kick os pés e pernas movimentamse para dentro, em relação à linha média do corpo. Estas fases ocorrem em oposição, enquanto a perna direita está realizando o inkick, a perna esquerda está realizando o outkick $^{4}$. A técnica do eggbeater promove força resultante propulsiva para cima, sendo utilizada no polo aquático pelos jogadores para mantê-los à tona em posição vertical durante a execução de habilidades, como chutes a gol, passes e bloqueios (3-7).

Segundo Sanders (1,5-6), são duas as habilidades usadas para elevar a parte superior do corpo no polo aquático, ambas envolvendo o uso da pernada de eggbeater. A alçada (salto vertical), em que a parte superior do corpo é direcionada para cima de maneira explosiva para alcançar altura máxima por um pequeno intervalo. $\mathrm{O}$ suporte ou apoio, durante o qual o corpo é mantido em uma posição elevada por um longo tempo.

A identificação dos parâmetros biomecânicos adotados na execução da pernada de eggbeater é um aspecto importante para avaliar e melhorar a técnica do atleta e, consequentemente melhorar seu desempenho na modalidade esportiva. Baseado nesses pressupostos, esta revisão de literatura tem como objetivos apresentar e discutir parâmetros cinemáticos e cinéticos relacionados à técnica de execução do eggbeater a fim de fornecer atualização sobre o tema a pesquisadores, treinadores e atletas.

\section{Métodos}

Para obter os trabalhos que foram revisados, utilizaram-se as palavras-chave eggbeater kick, water polo e technique, em inglês e português nas bases Scopus, Portal Periódico da CAPES e Google Acadêmico. A busca foi conduzida na tentativa de abranger a totalidade dos artigos publicados sobre o tema 
em periódicos e congressos, desta forma foram selecionados 19 estudos (no período de 1996 a 2016, ao passo que não foram encontrados estudos de anos anteriores) que apresentavam análises cinemáticas ou cinéticas do movimento em questão. Dos 19 estudos selecionados (15 artigos originais e quatro artigos de revisão), 14 artigos eram de revistas indexadas, dois artigos de anais de congressos e três trabalhos publicados em páginas eletrônicas que apresentavam relação direta com o tema desta revisão. Todos os artigos revisados foram classificados em três categorias: 1) que apresentassem objetivo de desccrição de parâmetros biomecânicos para avaliação de desempenho (artigos originais); 2) que apresentassem objetivo de descrição de parâmetros biomecânicos para análise técnica (artigos originais) e 3) que fizessem apenas descrição da técnica (artigos de revisão) (Figura 1).

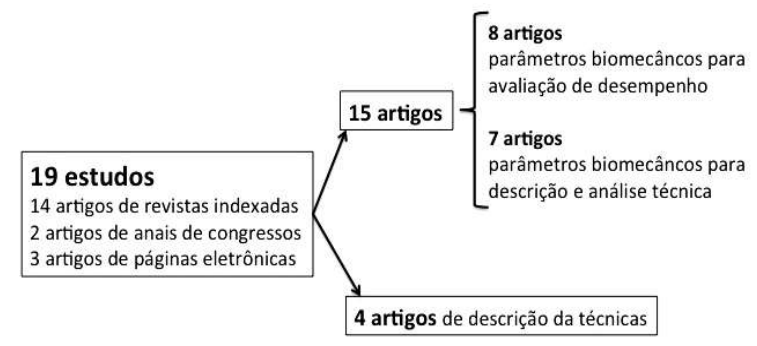

Figura 1 - Esquema dos estudos revisados

\section{Resultados e Discussão}

\section{Aspectos biomecânicos do meio líquido: conceitos básicos aplicados ao eggbeater}

Durante a execução do eggbeater, as pernas movem-se alternadamente, realizando movimentos circulares opostos, a perna direita move-se no sentido anti-horário e a perna esquerda no sentido horário (3). A água é um fluído que exerce forças sobre os corpos que se movimentam através dele, assim, é importante possuir uma compreensão sobre a mecânica dos fluidos. Algumas dessas forças tornam mais lenta a progressão dos corpos em movimento, outras auxiliam na propulsão (8).

Dois tipos de força são exercidos em um corpo por um ambiente fluido: o empuxo, causado pela sua imersão no fluido, e uma força dinâmica, relativa ao movimento entre corpo e fluído. A magnitude do empuxo é igual ao peso do volume de água deslocada pelo corpo imerso. O empuxo sempre atua verticalmente, para cima, em um objeto imerso. A força dinâmica é decomposta em dois componentes: arrasto e sustentação (9).

A força de sustentação atua perpendicularmente ao movimento relativo do corpo, sendo explicada pelo princípio de Bernoulli: os fluidos de movimento mais rápido exercem menor pressão do que os fluidos de movimento mais lento. No caso do eggbeater, quando o pé é movido através da água é criado um gradiente de pressão em cada lado do pé (o fluxo do líquido é mais rápido por cima do pé devido à sua forma, em seguida, uma área de baixa pressão é criada; se um sistema de baixa pressão existe acima do pé, e um sistema de alta pressão está localizado abaixo do mesmo, o pé será puxado para cima, área de menor pressão). Logo, esta força será transferida da parte inferior dos pés e pernas para todo o corpo, ajudando o jogador a ser suspenso ou elevado na água (7-9). Para Alexander and Taylor (7), a principal força que mantém o jogador suspenso na água é força de sustentação, causada pelo fluxo de água sobre o pé e a perna do atleta (Figura 2).

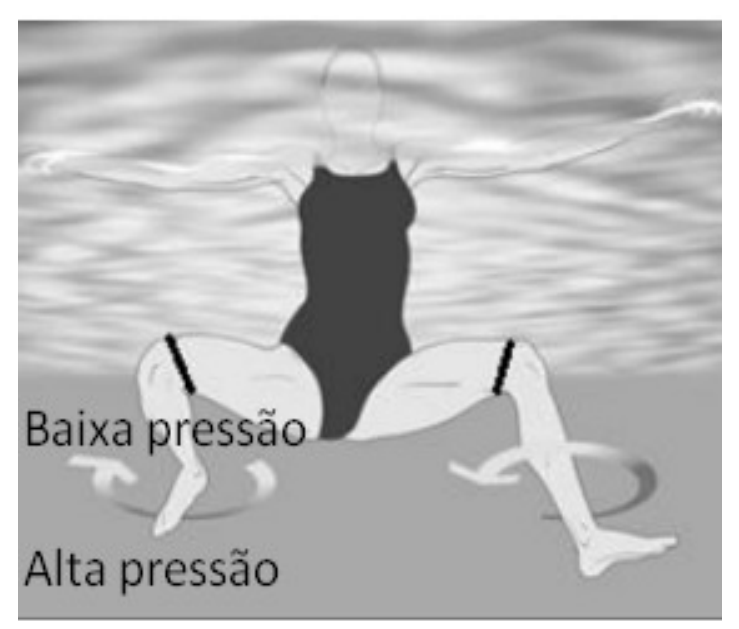

Figura 2 - Direção dos movimentos e zonas de pressão abaixo e acima dos pés

A força de arrasto é sempre oposta ao sentido da aplicação da força pelo corpo, por isso, se a mão empurra a água para trás, a força de arrasto atuará para impulsionar o corpo para frente. Durante o movimento do eggbeater o arrasto atua ao empurrar a água para baixo e a água empurra-o de volta, ajudando na sustentação do jogador (7-9). Segundo Alexander and Taylor (7), é 
provável que a propulsão no eggbeater resulte de uma combinação de ambos os componentes de sustentação e arrasto, bem como outras possibilidades, como o parafuso de Arquimedes.

Sanders (1), ao revisar e discutir a relação entre as forças de sustenção e arrasto concluiu que esta envolve um movimento dos pés similar a um palmateio (movimento realizado pelas mãos, com trajetória curvilínea, que promove sustentação e deslocamentos no meio líquido). $\mathrm{O}$ que, segundo o autor, sugere um papel importante à força de sustentação no que se trata do apoio do corpo do jogador em uma posição elevada em relação ao nível da água.

\section{Eggbeater: parâmetros biomecânicos para descrição e análise técnica}

Sanders ${ }^{5}$ investigou, por meio de cinemetria subaquática, as variáveis cinemáticas que contribuem para aumentar a altura da alçada em 16 jogadores de polo aquático (atletas novatos e experientes). As variáveis foram quantificadas por meio de análises cinemáticas tridimensionais. A medida da altura do vértex em relação ao nível da água foi usada para avaliar o desempenho. A mesma variou de 0,50 a $0,90 \mathrm{~m}$ e foi relacionada à orientação dos movimentos ântero-posterior e médio-lateral (plano horizontal) realizados pelos pés. A partir da análise de regressão múltipla, verificou-se que a velocidade do movimento do pé, a amplitude de extensão do joelho e o ângulo inicial do tronco (parte superior do corpo) em relação à horizontal, explicaram $74 \%$ da variância da altura máxima $\left(\mathrm{R}^{2}=0,744\right.$; $\mathrm{p}<0,01)$; todas em relação positiva com a altura máxima. Foi sugerido por Sanders (3) que a técnica envolve a utilização eficaz de ambas as forças, de arrasto e sustentação.

Ainda, Sanders (6) analisou a técnica de eggbeater com cinemetria tridimensional em 12 jogadores de polo aquático durante a execução de $30 \mathrm{~s}$ de eggbeater de suporte sem o auxílio das mãos (palmateio). Constatou-se que a altura do vértex, mantida durante o teste foi de 0,22 a 0,42 m (usada para classificar o desempenho). Os pés moveram-se em trajetórias curvilíneas, havendo contribuições substanciais dos movimentos nas direções vertical, ântero-posterior e médio-lateral. Os movimentos dos jogadores que atingiram maiores alturas apresentaram-se mais "arredondados" do que os dos jogadores com menos sucesso. Em particular, os jogadores experientes tinham seus movimentos mais focados no sentido ântero-posterior do que os novatos, isso ocorrendo notavelmente por manterem-se mais tempo próximos do ângulo máximo de flexão do joelho, enquanto que os jogadores com menor desempenho tiveram mudanças repentinas de direção. As variáveis de desempenho não são independentes umas das outras, isto é, jogadores que se mantiveram em boa altura foram caracterizados pelo movimento rápido dos pés, grandes movimentos no sentido ânteroposterior, e pequenos movimentos na direção vertical. Quando todas estas três variáveis foram incluídas em um modelo de regressão, foram responsáveis por $90 \%$ da variação da altura mantida no teste. Conclui-se que os jogadores precisam desenvolver a capacidade de manter altas velocidades na movimentação dos pés durante todo o ciclo da pernada.

O estudo de Homma and Homma (4) investigou a técnica de eggbeater de seis atletas de elite de nado sincronizado, dividindo-as em três duplas (excelente, bom e pobre, baseando-se em resultados nacionais) e classificou como "eggbeater do tipo horizontal" os movimentos das melhores atletas e, "eggbeater do tipo vertical" os movimentos das atletas menos qualificadas. Foi constatado que as melhores atletas faziam uma forte rotação interna do quadril durante a fase em que os pés e as pernas estão se movimentando para fora (out-kick), empurrando a água com a parte interna da coxa e da perna durante essa fase. Essa rotação interna do quadril é acompanhada por extensão do joelho, uma flexão plantar e pela abdução do tornozelo. $\mathrm{Na}$ outra fase do movimento, quando a perna e o pé se movimentam para dentro (in-kick), o ângulo de rotação interna do quadril é menor, ocorrendo a flexão do joelho, dorsiflexão e adução do tornozelo. Os resultados corroboram os achados dos estudos de Sanders (5-6).

Corrêa et al. (10) analisaram o chute a gol de seis atacantes a partir da marca do pênalti, 
enfatizando o movimento do eggbeater. Foram marcadas as articulações do quadril, joelhos, tornozelos e pés. Utilizou-se uma câmera subaquática para gravar, em vídeo, os movimentos no plano sagital. As variáveis avaliadas foram: deslocamento vertical do quadril do ponto inicial de preparação até a altura máxima atingida; ângulo de joelho das duas pernas, com ênfase no sincronismo; deslocamento e velocidade dos pés no plano sagital. O deslocamento do quadril variou de 0,15 a $0,29 \mathrm{~m}$ durante o chute a gol. O sincronismo está ligado à flexão e extensão do joelho e como os indivíduos diferem em termos de qual joelho estendem primeiro, em vez de se utilizar a denominação joelho direito ou esquerdo, foram utilizados, por Corrêa et al. (10), os termos joelho 1 e joelho 2 , relativos à primeira e à segunda extensão. $\mathrm{O}$ indivíduo que obteve o maior deslocamento no instante da impulsão final mudou $o$ sincronismo, adiantando a extensão do joelho 2 , que acontece logo após a extensão do primeiro, também apresentou uma maior amplitude na extensão de ambos os joelhos e maiores valores da velocidade horizontal do pé e adotou um padrão curvilíneo de movimento do pé. Os valores atribuídos à altura alcançada situaram-se abaixo dos descritos por Sanders (5), em que os atletas alcançaram de 0,50 a $0,90 \mathrm{~m}$ no impulso para $o$ arremesso. Um fator que dificulta a comparação entre os resultados é que os valores calculados por Sanders (5) referem-se à altura do vértex em relação à superfície da água, o que, em média, segundo o próprio autor, acrescenta um valor de $0,20 \mathrm{~m}$ ao valor da altura máxima alcançada.

Oliveira el al. (11) determinaram os padrões cinemáticos associados à produção de força vertical na execução da pernada de eggbeater. Para tal, foram avaliados 12 jogadores de polo aquático em nove ciclos de eggbeater quando foram obtidos os padrões que são responsáveis por maximizar a produção de força vertical no eggbeater, sendo estes: o movimento dos pés em alta velocidade no plano horizontal, grandes amplitudes de abdução e flexão dos quadris e rápidas extensão/flexão dos joelhos. Ainda, 81\% da variância da força vertical do eggbeater se explicam por estas três últimas variáveis.
Como referido em estudos anteriores, o movimento verticalizado dos pés foi associado negativamente à força vertical.

Ainda, Oliveira e Sanders (12) investigaram a lateralização motora dos membros dominante e não dominante na execução do eggbeater. Para tal estudo foram avaliados nove ciclos completos do movimento de 12 jogadores destros de polo aquático. Foi realizada análise tridimensional do eggbeater. Foram encontradas assimetrias nos padrões de força produzidas inter-membros para as fases equivalentes intra-ciclo. Baseando-se nos padrões de força vertical atingidos na realização do movimento, o tornozelo não dominante apresentou menor eficiência na fase de recuperação do eggbeater em relação à mesma articulação do membro dominante, sendo esta ineficiência uma consequência de maiores ângulos de inclinação negativos criados pelo pé não dominante onde, segundo os autores, esses ângulos de inclinação negativos neutralizam as forças de sustentação. Conclui-se que a maior eficiência do membro dominante, em relação ao não dominante, na fase de recuperação ocorreu devido às diferenças bilaterais entre-membros nos movimentos de rotação interna e externa e inversão das articulações dos quadris e tornozelos, respectivamente.

Contudo, sob fadiga, os padrões de movimento na execução da técnica do eggbeater apresentam alterações como as descritas por Oliveira et al. (13) que investigaram os efeitos da fadiga na técnica do eggbeater em 12 jogadores. Avaliaram força vertical, velocidade dos pés e ângulos dos quadris, joelhos e tornozelos em 27 ciclos de eggbeater, sendo nove ciclos em cada fase: inicial $(0 \%=$ sem fadiga $)$, metade do período de teste $(50 \%$ fadiga) e final do teste $(100 \%$ fadiga). A média da força vertical diminuiu progressivamente com o tempo. A velocidade dos pés reduziu em função da fadiga, aumentando a duração do ciclo. Os ângulos de abdução e flexão do quadril diminuiram com a fadiga, enquanto a rotação interna do quadril e a inversão do tornozelo aumentaram com a fadiga. A velocidade angular diminuiu para todas as articulações envolvidas. 
Eggbeater: parâmetros biomecânicos para avaliação de desempenho

Outros estudos preocuparam-se em definir e avaliar as características cinéticas e mecânicas e suas implicações na performance. O estudo de Dopsaj ${ }^{14}$ teve como objetivo definir as características cinéticas e mecânicas de $10 \mathrm{~s}$ máximos de eggbeater amarrado em 14 jogadores de elite de polo aquático. Foi utilizado o deslocamento para frente com as mãos fora da água. Foram realizadas as seguintes medidas (valores de uma pernada de eggbeater): duração, força máxima, média da força, impulso, taxa de produção de força e frequência de pernadas por minuto. Todos os dados foram descritos em valores absolutos e relativos à massa corporal. A análise de confiabilidade revelou-se altamente significativa em 96,81\%, com Coeficiente de Correlação Interclasse de 0,918, e, em medidas de média, de 0,994. Dentre todas as variáveis de análise, somente os valores de força máxima durante os $10 \mathrm{~s}$ de eggbeater se mostraram significativos para o desempenho.

O chute a gol é considerado um dos mais importantes aspectos do desempenho em vários esportes. No polo aquático, o chute a gol é uma das habilidades importantes que utiliza o eggbeater. Enfatiza-se a melhora da velocidade do chute a gol, pois o pressuposto é de que quanto mais rápido a bola é lançada, menos tempo o goleiro tem para desviar a bola, aumentando a probabilidade de marcar gols. Aceita-se que a elevação máxima do corpo da água é fundamentalmente relacionada com o desempenho, não só para arremessar a bola, como também para bloquear os chutes e passes dos adversários (6,15-16).

A obtenção de determinada altura fora da água permite que o atleta flexione o tronco anterior e lateralmente, obtendo a mecânica ideal para o chute. Além disso, quanto maior a altura atingida fora da água, menor será a resistência ao movimento exercida pelas forças de arrasto (menores no ar). Idealmente, o ponto mais alto da alçada ocorre no momento da liberação da bola. A altura aproximada de $76 \mathrm{~cm}$ acima da superfície da água é referenciada como sendo a altura que posiciona o lançamento da bola no ponto médio da altura da goleira (17).
Segundo Alexander et al. (17), o aspecto desafiador do chute a gol do polo aquático, em comparação com outras modalidades, é a falta de apoio firme em que as pernas possam se apoiar durante a produção de força do arremesso. A suspensão na água impede o atleta de transferir as forças de reação do solo através do corpo no momento do chute. A ação das pernas durante o chute a gol no polo aquático tem um propósito muito diferente do que em qualquer esporte jogado em terra. Elas assumem um papel de apoio, manutenção do equilíbrio e da altura da água, bem como contribuir para a produção de força.

Alguns estudos buscaram relacionar a potência dos membros inferiores com o desempenho da alçada, usando como indicador de potência o salto vertical em terra. O estudo de McCluskey et al. (16) objetivou determinar a existência da relação entre a velocidade de chute e a altura do salto vertical na água (alçada) em 22 jogadoras de alto nível do polo aquático. As variáveis analisadas foram: potência dos membros inferiores (por meio de salto vertical em terra), altura máxima do salto vertical na água (em qualquer momento do chute e no momento de liberação da bola), velocidade da bola durante o chute a gol e medidas antropométricas. Foram realizados seis chutes e analisados os três mais rápidos. A potência dos membros inferiores foi o preditor mais significativo para a velocidade máxima do chute apresentado por análise de regressão múltipla, sendo responsável por $62 \%$ da variância da velocidade máxima $(\mathrm{p}<0,001)$. Após controlar o efeito da potência, a altura máxima no momento da liberação da bola também foi significativa, responsável por $7 \%$ da variância da velocidade máxima $(\mathrm{p}=0,049)$. Conclui-se que as jogadoras com maior potência de membros inferiores são capazes de gerar maior elevação fora da água sendo capazes de chutar a bola com maior velocidade.

No entanto, Platanou (15) investigou a relação entre as alturas do salto vertical em terra e do salto vertical na água, em 43 jogadores de elite do polo aquático. $\mathrm{O}$ valor médio do salto vertical na água foi de $68,3 \pm$ $4,6 \mathrm{~cm}$, enquanto o valor médio do salto vertical em terra foi de $49,6 \pm 6,5 \mathrm{~cm}$. O coeficiente entre os dois saltos foi muito 
baixo $(\mathrm{r}=0,25)$, levando à conclusão de que o desempenho da impulsão vertical na água correlacionou-se mal com a capacidade explosiva dos membros inferiores que foi avaliada pelo salto vertical em terra.

Em estudo subsequente, Platanou (18) avaliou a precisão e a confiabilidade do teste de salto vertical na água, descrito no estudo anterior (11). Participaram do estudo 17 jogadores da Liga Grega que foram testados ao executar o salto vertical na água medido de duas maneiras distintas: 1) em laboratório com análise cinemática bidimensional (2D) e 2) com o teste de campo específico (salto vertical na água em eggbeater: máxima altura atingida em esforço máximo), com três tentativas para cada procedimento. Os resultados para o teste de campo foram: 67,6 $\pm 6,0 \mathrm{~cm}, 67,5 \pm 5,7 \mathrm{~cm}$ e $67,4 \pm 5,6 \mathrm{~cm}$ (para as três tentativas, respectivamente), sendo a média $68,6 \pm 5,4 \mathrm{~cm}$, com uma variação de 56,5 a $79,9 \mathrm{~cm}$. Por outro lado, o valor médio para o salto vertical na água com análise $2 \mathrm{D}$ em laboratório foi $65,3 \pm 5,9 \mathrm{~cm}$. Não foram encontradas diferenças na análise de variância com medidas repetidas entre as três tentativas do teste de campo e o melhor desempenho medido com a técnica 2D. O coeficiente de correlação intraclasse encontrado foi de $0,92 \mathrm{e}$ 0,98 entre os três saltos verticais do teste de campo, enquanto que a correlação entre a avaliação do melhor salto vertical do teste de campo e o melhor salto vertical avaliado pelo teste de laboratório foi 0,96 . Os resultados indicam que o teste de campo específico é um método confiável para avaliar a capacidade para o salto vertical na água.

Stirn el al. (2) examinaram diferentes testes para avaliar o eggbeater em 28 jogadores de polo aquático. Foram propostos um teste em terra (salto vertical) e 10 testes em água com a utilização do eggbeater. Conclui-se que os testes mais apropriados para avaliar o eggbeater foram os testes que apresentavam maior especificidade com situações de jogo. O estudo de Zinner et al. (19) teve como objetivos: 1) avaliar o eggbeater e o desempenho no chute usando testes específicos, 2) explorar a relação entre 0 eggbeater e o desempenho no chute e 3) investigar a relação entre o eggbeater e testes de força em laboratório. Foram realizados testes de força dinâmica e isométrica em laboratório para os grupos musculares comumente envolvidos na modalidade (adutores, abdutores, abdominais e peitorais), antropometria (estatura, massa e comprimento dos membros superiores) e seis testes na água, sendo estes: eggbeater endurance, eggbeater agilidade, velocidade máxima de chute, altura máxima do salto vertical, $400 \mathrm{~m}$ de nado (capacidade aeróbica) e sprint (capacidade anaeróbica). Os resultados apresentaram correlação entre a altura do salto vertical e o comprimento do braço $(\mathrm{p}<0,001 ; \mathrm{r}=0,89)$, também foram encontradas correlações entre a força máxima dinâmica dos grupos musculares avaliados e os resultados encontrados nos testes na água. Foram encontradas correlações significativas entre o eggbeater endurance e a altura máxima do salto vertical $(\mathrm{p}<0,001 ; \mathrm{r}=0,65)$ e eggbeater agilidade e a altura máxima do salto vertical $(\mathrm{p}<0,001 ; \quad \mathrm{r}=0,86) . \quad$ A relação entre $\mathrm{o}$ velocidade do chute e a altura do salto vertical apresentaram correlação moderada $(\mathrm{p}=0,04$; $\mathrm{r}=0,54)$. Concluiu-se que é necessário avaliar os atletas tanto dentro quanto fora da água devido à complexidade do perfil dos jogadores de polo aquático.

Embora não diretamente relacionados ao estudo das variáveis biomecânicas, Uljevic et al. ${ }^{20}$ construíram e validaram um protocolo de testes para determinar as capacidades condicionantes específicas do polo aquático, constituída de testes combinados para simular situações de jogo (sprint, chute, salto vertical e precisão). Os testes foram realizados de forma isolada e combinada (após exaustão) por 54 jovens jogadores, dentre eles a equipe nacional. $\mathrm{O}$ protocolo de testes combinados discriminaram qualitativamente os dois grupos de jogadores (equipe nacional vs atletas de equipes) de forma mais eficaz do que os testes isolados, provavelmente por representar melhor as exigências de desempenho em situação real de jogo.

Melchiorri et al. (21) aplicaram teste de eggbeater com incremento progressivo de carga até a exaustão utilizando um colete artesanal. Participaram dos testes 42 jogadores de polo aquático (20 amadores e 22 elite). O protocolo consistiu em diferentes tentativas até a exaustão (cargas de 5 a $25 \mathrm{~kg}$, 
com incremento de $2,5 \mathrm{~kg}$ em cada tentativa) e intervalo de 20 minutos entre elas. $\mathrm{O}$ grupo amadores realizou seis tentativas até a exaustão (até $17,5 \mathrm{~kg}$ ), já o grupo elite, oito tentativas (até $22,5 \mathrm{~kg}$ ). Foram encontradas diferenças para o tempo de exaustão $(\mathrm{p}<0,01)$ entre os dois grupos em todas as diferentes sobrecargas, sendo o tempo do grupo amadores sempre menor. Porém, neste estudo, nenhuma variável cinética ou cinemática, do eggbeater, foi avaliada.

\section{Pontos fortes e limitações do estudo}

Este estudo buscou listar e discutir questões relacionadas à biomecânica do polo aquático, de modo específico, do eggbeater. Assim indica a professores, treinadores e jogadores como a técnica pode ser incrementada. Embora popular, principalmente na Europa, não há, ainda, extensa produção científica na área, o que pode comprometer, ainda a compreensão dos gestos da modalidade.

\section{Conclusão}

O movimento do eggbeater não deve ser realizado verticalmente, mas horizontalmente, nas direções ântero-posterior e médio-lateral. Durante o movimento, os pés desenham círculos paralelos à superfície da água, gerando força de sustentação. Para minimizar a verticalização do movimento, os joelhos e tornozelos devem se manter sempre elevados e o mais distante possível uns dos outros e os calcanhares próximos aos quadris. Recomenda-se também que o movimento dos pés mantenha elevada velocidade.

Em relação às possibilidades de análise do eggbeater, por ser um movimento realizado nos três planos, e dentro da água, tecnologias avançadas são necessárias. Para uma melhor compreensão do gesto, em relação à cinemática, análise tridimensional, parece ser a melhor ferramenta. Já em relação à análise cinética, os dados encontrados permitem verificar que existe dificuldade para a obtenção das variáveis. A cinemetria parece ser eficaz quando utilizada para calcular a velocidade (da bola em chutes a gol; dos pés e pernas) e a aceleração dos segmentos, permitindo, então, com a aceleração, a estimativa das forças geradas pelo movimento. Porém, para a obtenção da força não se tem uma técnica acurada, sendo utilizada, muitas vezes, a potência dos membros inferiores como um indicativo da mesma. Assim, existe a necessidade de desenvolver tecnologias para melhor analisar os parâmetros cinéticos do eggbeater.

\section{Declaração de conflito de interesses}

Não houve nenhum conflito de interesses no presente estudo.

\section{Declaração de financiamento}

Não houve nenhum tipo de financiamento para o estudo.

\section{Referências}

1. Sanders R.H. Lifting performance in aquatic sports. ISBS - Conference Proceedings Archive, 16 International Symposium on Biomechanics in Sports. 1998.

2. Stirn I; Strmecki J, Strojnik V. The Examination of Different Tests for the Evaluation of the Efficiency of the Eggbeater Kicks. Journal of Human Kinetics. 2014;. 41: 215-226.

3. Sanders RH. Strength, flexibility and timing in the eggbeater kick. 2005. Internet: Acessado em 25/04/2016. https://isis.ku.dk/kurser/blob.aspx?feltid= 206584.

4. Homma M, Homma M. Coaching points for eggbeater kick in synchronized swimming based on three-dimensional motion analysis. Sports Biomechanics. 2005; 4(1): 73-88.

5. Sanders RH. A model of kinematic variables determining height achieved in water polo boosts. Journal of Applied Biomechanics. 1999; 15(3): 270-283.

6. Sanders RH. Analysis of the Eggbeater Kick Used to Maintain Height in Water Polo. J Appl Biomech. 1999;15(3):28491.

7. 7. Alexander M, Taylor $\mathrm{C}$. The technique of the eggbeater kick. 2008. Internet. Acessado em 25/04/2016. https://www.waterpolo.ca/admin/docs/LT AD/EggBeater.pdf. 
8. Hall SJ. Biomecânica Básica. $5^{\mathrm{a}}$ ed. Barueri, SP: Manole. 2009.

9. McGinnis, P.M. Biomecânica do Esporte e Exercício. Porto Alegre: Artmed, 2002.

10. Corrêa SC, Teixeira S, Júnior EGG. Análise biomecânica da pernada alternada no polo aquático. Revista da Educação Física/UEM. 2010; 21 (1): 13-23.

11. Oliveira N, Chiu CY, Sanders RH. Kinematic Patterns Associated with the Vertical Force Produced during the Eggbeater Kick. Journal of Sports Sciences. 2015; 33(16): 1675-1681.

12. Oliveira N, Sanders DRH. Kinematic and kinetic evidence for functional lateralization in a symmetrical motor task: the water polo eggbeater kick. Exp Brain Res. 2015; 233:947-957.

13. Oliveira N, Saunders DH, Sanders RH. The Effect of Fatigue-Induced Changes in Eggbeater-Kick Kinematics on Performance and Risk of Injury. International Journal of Sports Physiology and Performance. 2016; 11:141-145.

14. Dopsaj M. Pulling force characteristics of $10 \mathrm{~s}$ maximal tethered eggbeater kick in elite water polo players: a pilot study. Biomechanics and Medicine in Swimming. 2010; 11:69-71.

15. Platanou T. On-water and dryland vertical jump in water polo players. Journal Sports Medicine and Physical Fitness. 2005; 45(1): 26-31.

16. McCluskey L, Lynskey S, Leung CK, Woodhouse D, Briffa K, Hopper D. Throwing velocity and jump height in female water polo players: Performance predictors. Journal of Science and Medicine in Sport. 2010; 13:236-240.

17. Alexander M, Hayward J, Honish A. Water Polo: A Biomechanical Analysis of the Shot. 2011.

18. Platanou T. Simple 'in-water' vertical jump testing. Kinesiology. 2006; 38(1): $57-62$.

19. Zinner C, Sperlich B, Krueger M, Focke T, Reed J, Mester J. Strength, Endurance,
Throwing Velocity and in-Water Jump Performance of Elite German Water Polo Players. Journal of Human Kinetics. 2015; 45: 149-156.

20. Uljevic O, Esco MR, Sekulic D. Reliability, Validity, and Applicability of Isolated and Combined Sport-Specific Tests of Conditioning Capacities in TopLevel Junior Water Polo Athletes. Journal of Strength Conditioning and Research. 2014; 28(6): 1595-1605.

21. Melchiorri G, Viero V, Triossi $T$, Tancredi V, Galvani C, Bonifazi M. Testing and Training of the Eggbeater Kick Movement in Water Polo: Applicability of a New Method. Journal of Strength Conditioning and Research. 2015; 29(10): 2758-2764. 